\title{
APPROCHE SÉMIO-RHÉTORIQUE DU TEXTE ANIMÉ DANS LA PUBLICITÉ EN LIGNE
}

\begin{abstract}
Alexandra Saemmer ${ }^{1}$
Cet article se penche sur les « couplages " entre texte et mouvement dans les bannières et annonces publicitaires en ligne. Sera mobilisée une méthodologie que j'appelle « rhétorique de la réception ». Cette méthodologie a comme objectif d'identifier les procédés rhétoriques du texte numérique, d'étudier comment ils anticipent sur les pratiques de lecture, et d'énoncer des hypothèses sur la rencontre possible entre ces « potentiels d'action » (Iser) et les « horizons d'attente » (Jauss) individuels et socialement partagés du lecteur. L'approche est présentée de façon synthétique dans une première partie de l'article.

La rhétorique de la réception convoque un certain nombre d'éléments de l' " horizon d'attente » extra-textuel, constitué d'attentes, d'imaginaires et de représentations sociales que je propose d'appeler «figurations ». Même si ces figurations sont évolutives et peuvent varier d'une situation de lecture à une autre, la rhétorique de la réception adopte l'hypothèse que le lecteur s'inscrit dans des « communautés interprétatives » (Fish). Une deuxième partie de l'article est consacrée à l'identification de figurations courantes de la publicité en ligne. Émerge la possibilité d'une "persuasion sans conscience », mobilisée et renforcée par la mise en mouvement de certains éléments textuels.

Un corpus de bannières publicitaires et d'annonces commerciales est présenté dans une troisième partie. J'avance l'hypothèse que certains mouvements agissent sur le lecteur en tant qu' « icônes », mettant en place une « résonance mimétique » qui idéalement
\end{abstract}

1 Alexandra Saemmer est Professeure en Sciences de l'Information et de la Communication à l'Université Paris 8 Vincennes/Saint-Denis.

Recherches en communication, $\mathrm{n}^{\circ} 39$ (2013). 
renforce le message textuel, mais peut aussi agir sur le lecteur indépendamment du texte.

Les textes sont néanmoins bien présents dans ces exemples. Couplés aux mouvements, ils forment des " procédés rhétoriques ", fondés sur l'interaction entre le texte linguistique et l'animation. J'identifierai dans une dernière partie les « figures de la lecture » qui émergent à partir de ces couplages entre textes et mouvements.

La méthodologie et les résultats de cette étude du texte animé renvoient aux problématiques d'une " sémiotique du sensible», mise en œuvre depuis plusieurs années dans d'autres domaines. Les enjeux de cette relation sont brièvement discutés en guise de conclusion.

Tout visiteur de sites web est régulièrement confronté à des éléments publicitaires. Dans ces annonces qui prennent parfois la forme de bannières, le texte est fréquemment animé par toutes sortes de mouvements : du clignotement, des passages de gauche à droite ou de haut en bas, de l'effacement... Affirmer que ces mouvements sont mobilisés pour «attirer l'attention » du lecteur est un allant de soi. Dans cet article, il s'agira de se pencher sur les « couplages » entre textes et mouvements dans la publicité en ligne afin de circonscrire leur « potentiel d'action » avec plus de précision. Je mobiliserai une méthodologie sémio-rhétorique que j'ai appelée ailleurs « rhétorique de la réception $»^{1}$, et qui sera brièvement présentée dans une première partie. Je mettrai ensuite cette méthodologie en œuvre à travers l'analyse des animations textuelles dans trois bannières et annonces publicitaires.

\section{La « rhétorique de la réception » du texte numérique}

La rhétorique, art de la persuasion et de la délibération, art poétique et art de la manipulation, propose par le biais de ses « figures » une restructuration du réel adressée à un public. La « rhétorique de la réception du texte numérique » que j'essaie de mettre en place depuis plusieurs années, a comme objectif d'identifier les procédés rhétoriques du texte numérique, d'étudier comment ils anticipent sur les pratiques de lecture, et d'énoncer des hypothèses sur la rencontre possible entre

1 Cet article résume et retravaille des recherches présentées dans mon mémoire d'habilitation (juin 2013). 
ces « potentiels d'action » et les « horizons d'attente » individuels et socialement partagés du lecteur.

Cette méthodologie s'inspire des théories de la réception (Iser, 1976 ; 1995 ; Jauss, 1978 ; 2010), de la Nouvelle Rhétorique qui repositionne l'auditoire au centre du processus de communication (Perelman, 1977), des théories de 1' « énonciation éditoriale » (Souchier, 1998), et de la pensée de la « trivialité » (Jeanneret, 2008) qui étudie les caractéristiques sémiotiques d'un objet culturel et sa circulation sociale. À partir de ces fondements théoriques, la rhétorique de la réception du texte numérique met en place une approche articulée autour de ce que je propose d'appeler les « figures de la lecture ».

Les « figures de la lecture » anticipent sur les pratiques à travers les « répertoires » du texte (les conventions sociales qui y sont évoquées, les allusions aux événements contemporains et historiques, donc tous ces éléments qui fondent la situation de communication entre le texte et le lecteur selon Iser ,1976), et différents procédés rhétoriques spécifiques au texte numérique. Ces procédés reposent par exemple sur le potentiel d'action du rapport de contiguité entre deux ou plusieurs textes établi par un hyperlien, ou sur le couplage entre les signes alphabétiques et le mouvement dans le texte animé. Les « formes-modèles » (Jeanneret, Souchier, 2005) comme la police de caractères, la mise en espace du texte, ou le mouvement agissant sur le lecteur avant et pendant le déchiffrage d'un texte animé, préfigurent également un certain nombre de pratiques de lecture.

L'acte de lecture lui-même est défini comme un champ de tensions entre ces potentialisations opérées par les formes et figures du texte numérique, et leur actualisation forcément partielle par le lecteur dans une situation précise. La rhétorique de la réception convoque ainsi des éléments de l'« horizon d'attente » (Jauss) extra-textuel, constitué d'attentes, d'imaginaires et de représentations sociales que je propose d'appeler «figurations ». Même si ces figurations sont évolutives et peuvent varier d'une situation de lecture à une autre, la rhétorique de la réception part de l'hypothèse que le lecteur s'inscrit dans des « communautés interprétatives » (Fish, 1980).

\section{Figurations de la publicité en ligne}

Quelles pourraient être des figurations partagées des bannières et annonces publicitaires sur Internet ? D'après Laurent et Kapferer (1986), l'effort du lecteur consiste avant tout à ne pas lire ces 
éléments. L'intérêt pour la publicité en ligne est faible, malgré un poids économique important. Selon une étude menée par McKinsey pour IAB Europe $^{1}$, les annonceurs européens ont consacré en 2009 14,7 milliards d'euros à la pub en ligne. $70 \%$ des usagers de l'Internet interrogés dans cette étude, attribuent suffisamment de valeur à la gratuité des services offerts grâce à la pub pour l'accepter comme un " mal nécessaire » : ils semblent cependant penser que leur faible intérêt pour la publicité permet d'échapper à son influence.

La moyenne du taux de clic sur la publicité en ligne a effectivement toujours été faible. En 2006, elle est passée à $0,15 \%$, et la tendance est à la baisse. Selon une étude d'eMarketer en $2010^{2}$, certains professionnels ont pourtant pris conscience du fait que l'influence de la pub sur le lecteur ne se mesure pas seulement par le " passage à l'acte »du clic. Les bannières, souvent placées en haut de l'écran, entrent dans le champ de vision «fovéale » situé à un angle compris entre $0^{\circ}$ et $2^{\circ}$ du point de focalisation visuelle. Shapiro et Krishnan (2001) ont mobilisé des recherches sur la " mémoire implicite » pour montrer l'« efficacité » de certaines bannières, même lorsque l'attention du lecteur n'est pas focalisée dessus. Didier Courbet, Amélie Borde et al. (2004) ont poursuivi les recherches sur cette persuasion "sans conscience ", montrant que le récepteur peut être amené à effectuer un acte d'achat suite à une exposition même furtive à une publicité.

Plusieurs hypothèses peuvent être avancées pour expliquer cette «persuasion sans conscience » potentiellement mobilisée par les bannières : le lecteur reconnaît peut-être une marque grâce à ses couleurs et formes même s'il ne prête qu'une attention réduite à la bannière. Et les mouvements dans les bannières animées l'« atteignent » peut-être de façon autrement sensible que le texte statique, même si ce texte n'est pas déchiffré.

Je propose effectivement l'hypothèse que certains mouvements présents dans les bannières et annonces publicitaires peuvent agir sur le lecteur en tant qu' " icônes ", mettant en place une " résonance mimétique » qui peut influencer le lecteur indépendamment du texte, constituant des « figures de la lecture » sur la page-écran. Les textes à

1 « 100 milliards d'euros de services internet financés par la pub en ligne ». Rapport par IAB Europe, http://www.iabfrance.com/getfile.php?id=2833 (consulté le 26 mai 2012).

2 eMarketer. « Is the Click Still King? », http://www.emarketer.com/go/IAB_France. aspx (consulté le 26 mai 2012). 
lire sont néanmoins bien présents dans beaucoup de bannières animées. Couplés aux mouvements, ils forment des "procédés rhétoriques » fondés sur l'interaction entre le texte linguistique et l'animation. J'identifierai les « figures de la lecture » qui émergent à partir de ces couplages.

La focalisation directe ou indirecte sur une bannière publicitaire dépend de la « décision sémiotique » (Klinkenberg, 1996, p. 83) du lecteur, qui isole une partie intelligible du monde et en retire une signification. L'auteur-concepteur de bannières anticipe sans doute souvent sur les deux situations de " lecture » : celle où la bannière agit sur le lecteur sans qu'il y prête attention, et celle où la bannière se trouve lue et regardée de près. Dans cette deuxième situation de réception, l'anticipation des pratiques de lecture peut aller jusqu'au " partage poétique » de scènes imaginaires, comme l'a formulé Nicole Pignier (2005, p. 532). La prépondérance statistique d'un lecteur inattentif n'exclut pas la possibilité que certains lecteurs se laissent « prendre au jeu » d'une figuration plus onirique, poétique ou ludique de la publicité.

\section{Potentiels d'action de l'animation textuelle Les unités sémiotiques temporelles}

Le texte alphabétique présent dans une bannière publicitaire ou annonce commerciale sur internet mobilise des « répertoires » (Iser 1976 ; 1995) qui font appel à un savoir socioculturel partagé et fondent une partie de son " potentiel d'action ». Des animations comme le clignotement d'un mot, sa disparition ou son passage de gauche à droite, couplent un déroulement temporel au texte alphabétique. Comment circonscrire le « potentiel d'action » d'un tel mouvement ? Comment cette forme-modèle anticipe-t-elle sur les réactions du lecteur?

Comme je l'ai déjà dit, je considère certains mouvements comme des " icônes " qui s'approchent, par leur structure visuellement percevable, d'une représentation de leur référent d'expérience, faisant donc appel à des expériences que le lecteur a déjà rencontrées ailleurs. L'icône est ainsi définie comme un signe "motivé par ressemblance » (Klinkenberg, 1996, p. 377). Ce n'est pourtant pas forcément chaque mouvement seul, par exemple une apparition ou une disparition au sein d'un clignotement, qui fait appel à ce genre d'expériences déjà rencontrées. Ce sont plutôt le rythme et la vitesse qui semblent jouer un rôle prépondérant dans cette reconnaissance, comme j'ai pu l'observer à partir d'une étude comparative d'une cinquantaine de bannières 
et annonces publicitaires en ligne effectuée dans le cadre du projet « Signes et figures $»^{1}$.

En effet, des mouvements aux caractéristiques visuelles parfois très différentes peuvent se trouver couplés aux mêmes textes et semblent anticiper ainsi sur des pratiques de lecture semblables. Certains auteursconcepteurs font par exemple clignoter rapidement le mot « soldes »; d'autres le font s'agrandir et rétrécir au même rythme ; d'autres encore le font changer rapidement de couleur. Ce qui rapproche ces mouvements, malgré les différences dont je ne nie pas l'importance dans le processus de communication, est le rythme rapide et la répétition. La modélisation ci-contre à partir du mot « soldes » que j'ai fait clignoter, changer de couleur, gonfler et rétrécir au même rythme sur un même fond, a renforcé l'hypothèse que ces trois mouvements sont porteurs d'un potentiel d'action semblable, qui est reconnu par le lecteur grâce à des caractéristiques temporelles communes.

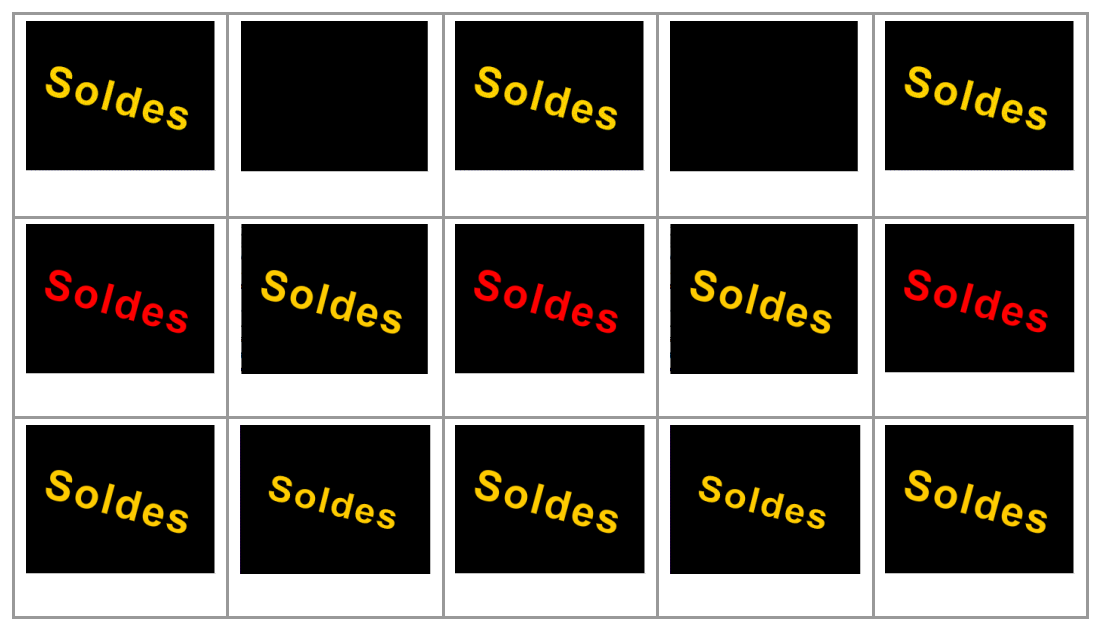

Figure 5. Correspondants visuels possibles de l'unité sémiotique temporelle « obsessionnel ». Le mot « soldes » change de couleur ou de taille à un rythme rapide etdefaçon réitéréesurces trois bannières quipourraientse trouversurun site commercial. Modèles construits à partir d'exemples du corpus, consultables aux adresses http://www.alexandrasaemmer.fr/corpus/obsessionnel/soldes1.html, http://www.alexandrasaemmer.fr/corpus/obsessionnel/soldes2.html, http:// www.alexandrasaemmer.fr/corpus/obsessionnel/soldes3.html.

1 Projet « Signes et figures de la création numérique », initié en 2009, avec Philippe Bootz, Serge Bouchardon, Jean Clément et Alexandra Saemmer. Ouvrage en préparation. 
À partir de l'étude du corpus, j'ai pu regrouper d'autres mouvements ou enchaînements de mouvements aux détails visuels parfois assez divergents, mais au potentiel d'action commun.

Par exemple, certains objets textuels " disparaissent " soit en s'effaçant lentement, soit en devenant de plus en plus flous. Les deux mouvements ont en commun une extinction progressive de l'énergie.

Certains éléments textuels s'agrandissent sur l'écran et donnent l'impression de se rapprocher progressivement ; d'autres « apparaissent » en devenant de moins en moins flous ; d'autres encore changent progressivement de position. Malgré les différences, ces mouvements décrivent une évolution linéaire sans perte d'énergie.

Pendant que j'effectuais ces regroupements, $\mathrm{j}^{\text {' }}$ ai pris connaissance ${ }^{1}$ des recherches sur les "unités sémiotiques temporelles » (UST) identifiées dans la musique par le MIM, un laboratoire de musicologie à Marseille ${ }^{2}$. Des expérimentations empiriques (Frey/Tijus/Poitrenaud, 2010) effectuées à partir des UST laissent penser que les auditeurs reconnaissent ces unités grâce à un certain nombre de caractéristiques touchant souvent au rythme, à la répétition et à l'intensité sonores. Une terminologie a été proposée par le MIM pour circonscrire ces caractéristiques.

L'unité « obsessionnel » est ainsi caractérisée par un enchainement de sons dont la répétition crée une pulsation rapide : par exemple une même note de piano répétée à plusieurs reprises. Des exemples sonores de l'« obsessionnel» sont accessibles sur le site du MIM³.

L'unité « sur l'erre » comporte une décroissance d'intensité sonore jusqu'à la disparition. Dans l'exemple proposé sur le site, le volume d'un son baisse lentement jusqu'à devenir inaudible ${ }^{4}$.La « trajectoire »

1 Grâce à la collaboration avec mon collègue Philippe Bootz autour du projet de recherche "Signes et figures de la création numérique ».

2 Laboratoire Musique et Informatique de http://www.labo-mim.org/site/index.php?2008/08/22/44-liste-des-19-ust (consulté le 13 mai 2012).

3 « Obsessionnel », http://www.labo-mim.org/site/index.php?2008/08/22/36obsessionnel (consulté le 15 mai 2012).

4 « Sur l'erre », http://www.labo-mim.org/site/index.php?2008/08/22/45-sur-1-erre (consulté le 15 mai 2012). 
est une unité qui présente une évolution linéaire. Elle est exemplifiée par un son qui n'en finit pas de monter ${ }^{1}$.

Lorsqu'on visualise maintenant les mouvements de clignotement, de changement de taille ou de changement de couleur rapides du mot " soldes » modélisés plus haut, et que l'on écoute en même temps l'extrait sonore d'un « obsessionnel » où une même note de piano est répétée de façon insistante, l'impression de synesthésie est frappante.

Il en est de même pour les exemples de bannières publicitaires sur lesquels un texte s'efface lentement, et le " sur l'erre » sonore où une note de piano s'éteint progressivement.

De même, la «trajectoire » dans laquelle un son n'arrête pas de monter, semble pouvoir entrer en correspondance avec les mouvements de déplacement ou de rapprochement progressifs d'un objet visuel sur l'écran.

Émerge donc l'hypothèse que les " unités sémiotiques temporelles » sont implémentables dans du son, du texte ou de l'image. La reconnaissance d'une telle unité par le lecteur semble fondée sur des processus d'intégration et de stabilisation d'expériences antérieures. Le lecteurreconnaît donc un «obsessionnel» parce qu'ill'a déjà expérimenté ailleurs, en observant par exemple le clignotement de boutons d'alarme. Il reconnaît l'unité " sur l'erre " grâce à des expériences de disparition et d'effacement, en observant par exemple une tâche d'eau s'évaporer. Il a expérimenté une " trajectoire » à chaque fois que, par exemple, une voiture s'est approchée de lui ou a traversé son champ visuel.

Le potentiel d'action des " unités sémiotiques temporelles » ne se démarque guère de ces référents d'expérience. L'« obsessionnel » peut ainsi renvoyer à des idées de danger ou d'urgence. Le "sur l'erre » peut renvoyer à des idées de perte et de disparition définitives. Une « trajectoire » peut renvoyer à des idées de matérialisation rassurante, de transformation sans perte de matière.

En circonscrivant ainsi le potentiel d'action d'un mouvement, il ne faut pourtant pas oublier que l'icône n'est pas seulement un objet pour la pensée consciente, mais surtout « une forme avec laquelle le corps percevant entre en résonance mimétique » (Meunier, 2006, p. 137). Grâce à son caractère insistant, l'obsessionnel semble ainsi pouvoir provoquer la capture de l'attention du lecteur indépendamment du texte précis auquel il est couplé. C'est sans doute la raison pour laquelle le

1 «Trajectoire », http://www.labo-mim.org/site/index.php?2008/08/22/28-trajectoireinexorable (consulté le 15 mai 2012). 
clignotement est si fréquemment utilisé dans les bannières publicitaires sur Internet, mais aussi dans la signalisation routière.

\section{Irradiations iconiques dans le texte animé}

Dans un texte animé, l'unité sémiotique temporelle se trouve couplée à des textes et, en fonction de l'horizon d'attente du lecteur, entre avec les répertoires de celui-ci dans une relation de contiguïté plus ou moins forte. Cette relation, fondée sur ce que je propose d'appeler une « irradiation iconique » d'un énoncé de mouvements sur le texte alphabétique, constitue des procédés rhétoriques qui anticipent de diverses manières sur les pratiques, constituant donc ce que j'appelle des « figures de la lecture ».

Je reviens à l'exemple du mot « Soldes » qui clignote sur un site commercial. La période des soldes en France s'étend sur un laps de temps assez court. Un sentiment d'urgence fait donc potentiellement partie des répertoires partagés entre le texte et le lecteur. L'iconicité du mouvement de clignotement peut renforcer ce sentiment d'urgence, lui conférant une « matérialité » que le texte seul ne possède pas.

Je propose maintenant d'identifier les « figures de la lecture » dans trois autres exemples de bannières et annonces publicitaires extraites du corpus constitué pour le projet « Signes et figures », et j'analyserai leur potentiel d'action.

\section{Présentation d'exemples de bannières publicitaires et d'annonces commerciales}

Le premier exemple a été sélectionné dans une « collection » de bannières publiée par le journaldunet.com. Le site présente les bannières sous forme de captures d'écran animées, séparées du contexte de la page-écran. Ce contexte peut néanmoins être partiellement reconstruit à partir d'indications données. Les deux autres exemples sont ce que j'appelle des « annonces publicitaires » : contrairement aux bannières, elles font partie du contenu principal de la page-écran.

L'une de ces annonces provient d'un site qui recense des casinos en ligne. Chaque casino est représenté par deux carrés : le premier contient des informations factuelles, le deuxième accueille le logo du 
site en question. Pour le « Casino Glamour » $»^{1}$, la somme « offerte » au joueur lors du premier enregistrement occupe une grande partie du carré. L'énoncé « 50 \$ offerts » s'affiche en violet. Un liséré blanc apparaît et disparaît rapidement autour de cet énoncé de sorte que se crée un mouvement de pulsation, tournant en boucle. Au-dessus de l'animation est inséré le logo du casino ; en-dessous se trouvent les injonctions « Jouez avec votre argent » et « Jouez maintenant ».

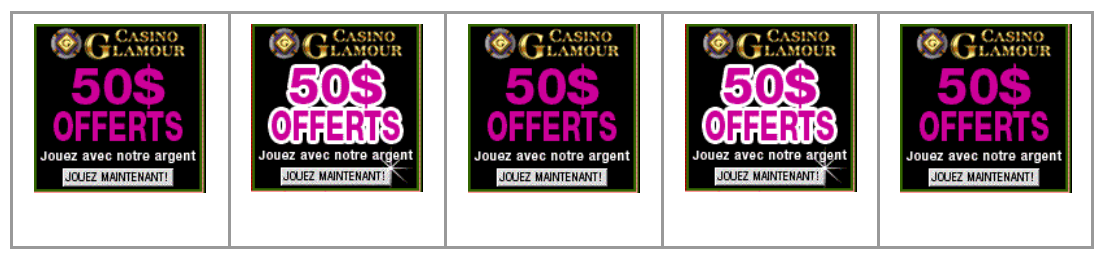

Figure 19. Capture d'écran d'un extrait du site Agentdunet http://www. agentdunet.com/casino.html (consulté le 15 juin 2012). Un liséré blanc apparaît et disparaît de façon rapide et réitérée autour de l'énoncé « 50 \$ offerts ».

La bannière suivante ${ }^{2}$ a été créée par l'agence Fullsix ${ }^{3}$ pour le Club Med. À droite s'affiche le portrait d'un skieur souriant. Sur ses lunettes se reflète une mosaïque de plusieurs images : un skieur sur la piste, deux personnes nageant dans une piscine, un bâtiment éclairé, un panorama alpin. Un tel panorama s'affiche également à côté du skieur. En haut à gauche s'y superpose le logo du Club Med. Le bas de la bannière est occupé par une banderole rouge sombre. L'énoncé « Le tout compris, c'est... » s'y affiche, juxtaposé à une petite flèche. Dès que le curseur effleure la banderole, un « expand» se déploie et détaille l'offre. Trois textes apparaissent et disparaissent successivement et lentement par « floutage » et " défloutage »: « Partez entre le 10 janvier et le 26 avril $09 »$, « 200 euros offerts à deux* (*voir conditions sur le site »), «Réservez avant le $1^{\text {er }}$ mars 09 ».

1 http://www.agentdunet.com/casino.html (consulté le 15 juin 2012).

2 http://www.journaldunet.com/ebusiness/rubriques/creations-pub-selectionsmensuelles/090306-selections-pub-fevrier/3.shtml (consulté le 15 juin 2012).

3 http://www.fullsix.com (consulté le 15 juin 2012). 


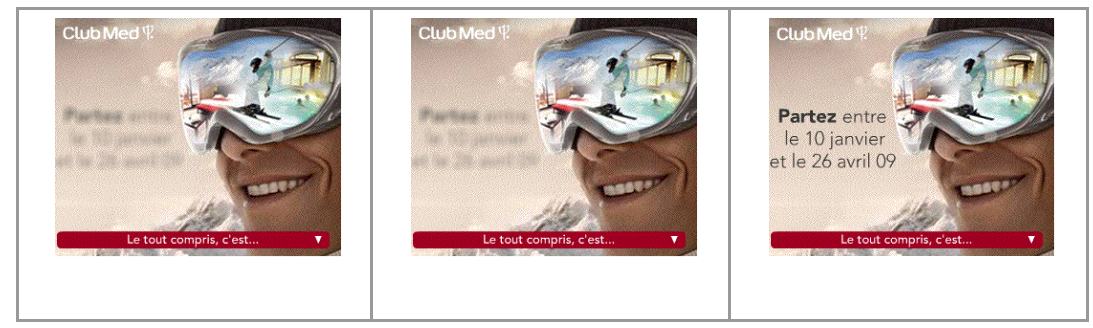

Figure 21. Bannière publicitaire publiée dans une collection de journaldunet http://www.journaldunet.com/ebusiness/rubriques/creations-pub-selectionsmensuelles/080527-selections-pub-mai/1.shtml (consulté le 15 juin 2012). L'énoncé « Partez entre le 10 janvier et le 26 avril 09 », d'abord flou, apparaît progressivement, se stabilise pendant quelques instants avant de redevenir flou.

La deuxième " annonce publicitaire » a été repérée sur le site des Parfums Kenzo ${ }^{1}$, mais n'est pas visible actuellement (avril 2013). Je la rends donc accessible sous forme de captures d'écran statiques et animées (voir encadré ci-contre pour l'adresse de consultation). L'annonce s'étale sur une grande partie de la page-écran. Dans un rectangle blanc "s'approchent» des noms de parfum jusqu'à passer hors-champ. La couleur des polices ne perd jamais son intensité lors de ce passage. Une fois disparus dans le hors-champ, les noms resurgissent du centre de l'annonce. À part quelques exceptions, ces noms s'affichent en noir. Le mouvement des mots est accompagné de bruits de pas qui s'approchent sur un fond sonore indistinct (bruits d'une ville, bruit de vagues ?). Une voix de femme chuchote « Il était une fois... Kenzo ». Les pas s'éloignent. Reste le léger bruitage accompagné d'une musique lente. Si le curseur effleure les mots, l'orientation de leur mouvement change légèrement. L'activation de chaque nom de parfum par clic mène vers un micro-site.

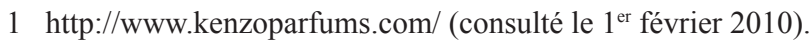




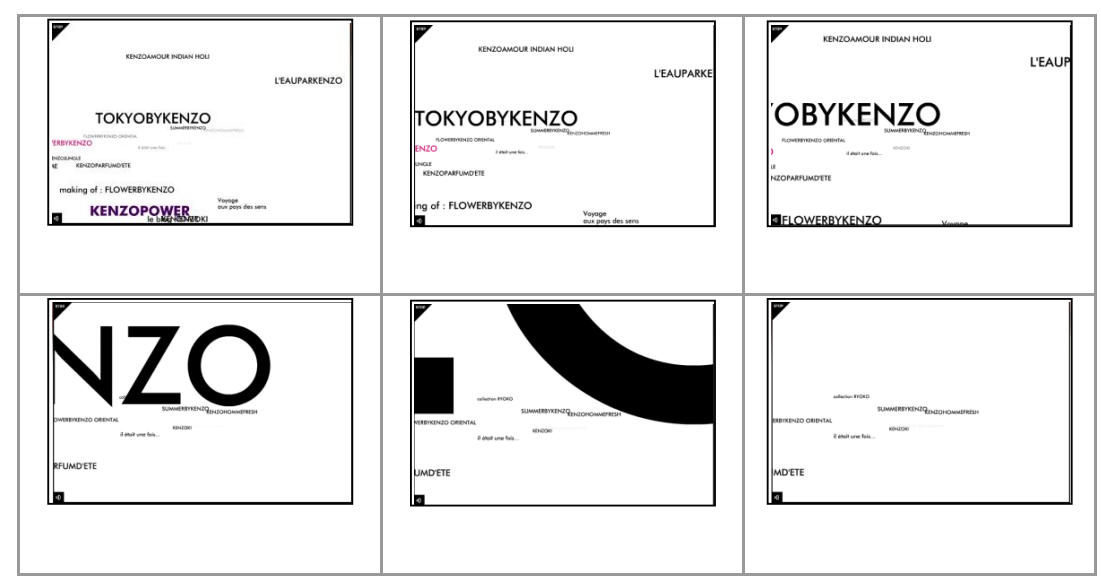

Figure 22. Capture d'écran d'un extrait du site Kenzo parfums qui n'est plus visible à l'heure actuelle (2012), et que je mets donc à disposition sous forme de capture d'écran http://www.alexandrasaemmer.fr/corpus/trajectoire/ kenzo.mov. Les noms de parfum changent progressivement de taille, donnant l'impression de s'approcher du lecteur.

\section{Formes-modèles du texte animé}

Dans les trois exemples, des textes ont été couplés à des mouvements, formant des procédés rhétoriques qui anticipent sur les pratiques du lecteur. Lorsqu'il entame effectivement la lecture des textes, un processus d' « irradiation iconique » peut donc se mettre en place (voir analyses plus loin). Cependant, les mouvements peuvent aussi « agir » sur le lecteur même s'il ne s'engage pas dans la lecturedéchiffrage. En tant qu'éléments visuels, ils orientent l'attention et les attentes du lecteur et créent potentiellement des sensations et émotions.

\section{«Obsessionnel »}

Dans l'annonce commerciale de "Casino Glamour », le liséré blanc autour de l'énoncé « $50 \$$ offerts », apparaissant et disparaissant rapidement, renvoie à l'unité sémiotique temporelle « obsessionnel». Je rappelle que le lecteur reconnaît l'obsessionnel parce qu'il a déjà vu clignoter des éléments dans son entourage : des boutons d'alarme, par exemple. Le potentiel d'action de cette icône peut donc se circonscrire par idées de stress, de nervosité, d'urgence, d'excitation émotionnelle, 
ou disons plutôt : il peut créer de telles émotions et sensations chez le lecteur, même si celui-ci ne lit pas le texte couplé au mouvement.

\section{« Trajectoire »}

Dans 1'《 annonce commerciale » des Parfums Kenzo, un mouvement continu anime les noms de parfum et s'apparente à une « trajectoire » grâce au caractère continu de l'animation, dont le lecteur peut supposer qu'elle se poursuit indéfiniment hors champ.

Sur la bannière du Club Med, les injonctions comme « Partez entre le 10 janvier et le 26 avril 09 », d'abord floues, deviennent progressivement lisibles. Ce mouvement s'apparente également à une " trajectoire ». Cette unité sémiotique temporelle peut renvoyer à des idées de transformation ou de dynamisme constants sans perte de matière et d'énergie, qui rassure potentiellement le lecteur par sa continuité.

\section{«Sur l'erre »}

Dans la bannière pour le Club Med, les énoncés se stabilisent pour quelques instants, puis redeviennent flous. Ce mouvement peut être considéré comme un correspondant visuel de l'unité sémiotique temporelle « sur l'erre », caractérisée par une extinction progressive de l'énergie et renvoyant à des idées de perte et de disparition inexorables. Sans lire les textes couplés à cette unité sémiotique temporelle, le lecteur éprouve potentiellement un sentiment de tristesse ou de frustration en percevant leur floutage.

\section{Figures de la lecture}

Les « couplages » entre textes et mouvements dans les bannières et annonces commerciales du corpus constituent des anticipations de lectures, qui s'actualisent de façon plus ou moins complète dans une situation de réception. La rhétorique de la réception a d'une part comme objectif de relever les procédés rhétoriques formés par ces couplages. D'autre part, elle énonce des hypothèses sur la rencontre possible entre ces procédés et l'horizon d'attente potentiel du lecteur. Dans le cas de la publicité en ligne, l'auteur d'une animation textuelle ne peut certainement pas compter sur le fait que le lecteur y prête une attention consciente. Couplant des textes à des mouvements, les auteurs des 
exemples sélectionnés anticipent néanmoins sur différentes pratiques de lecture possibles, dont une lecture attentive et concentrée. Se créent, entre textes et mouvements, des relations qui participent à fonder les conditions de réception des bannières, et pour lesquelles je propose cicontre une terminologie.

\section{Figure de la lecture «pro-affirmative»}

Bien évidemment, toutes les bannières et annonces expérimentant avec l'animation textuelle, ont comme objectif d'attirer l'attention du lecteur et de le convaincre. Certains couplages rhétoriques mettent cependant tout particulièrement à profit le potentiel d'action des formes-modèles pour souligner le potentiel d'action du texte, faisant appel à un lecteur qui se laisse convaincre par le caractère doublement affirmatif du message. Voilà pourquoi je propose de parler de figures d'une lecture « pro-affirmative ».

Dans l'annonce commerciale pour « Casino Glamour », l'énoncé « 50 \$ offerts » renvoie à l'idée d'un « cadeau » pécuniaire, créant potentiellement un sentiment d'excitation et de convoitise chez le lecteur. Cette excitation se trouve soulignée par la pulsation rapide, « obsessionnelle » du liséré blanc autour de l'offre. Je propose de parler plus précisément d'un " couplage emphatique ». Inséré dans une publicité pour un Casino en ligne, le mouvement peut par ailleurs rappeler les enseignes clignotantes dans les salles de jeux. Juxtaposé aux injonctions « Jouez avec votre argent » et « Jouez maintenant », le couplage peut renforcer l'envie de passer rapidement à l'acte, et préfigure un lecteur qui se laisse prendre au jeu d'une double affirmation, textuelle et émotionnelle.

Dans la bannière pour l'opérateur Club Med, les énoncés « Partez entre le 10 janvier et le 26 avril 09 » et « 200 euros offerts à deux* (*voir conditions sur le site) »s'affichent d'abord de façon floue, puis deviennent progressivement lisibles. Correspondants visuels de l'unité sémiotique « trajectoire », ils renvoient potentiellement à des idées de matérialisation, de révélation et de stabilisation progressives. Au bout de quelques instants, l'énoncé redevient pourtant flou, décrivant un mouvement « sur l'erre » potentiellement associé à des idées de perte et de disparition inexorables.

Le premier énoncé textuel précise la limitation de l'offre dans le temps. Le deuxième énoncé renvoie à l'idée d'une réduction en cas de voyage à deux. La combinaison de la «trajectoire » et du «sur 
l'erre » couplée aux énoncés pourrait souligner de façon sensitive à quel point l'offre est limitée dans le temps : elle se matérialise pendant quelques instants, puis elle est retirée du champ de vision, anticipant potentiellement sur les émotions de perte et de frustration d'un lecteur qui n'aura pas su saisir l'offre.

J'appelle plus précisément « couplage additionnel » une combinaison entre texte et icône, où les potentiels d'action non seulement se renforcent autour d'une idée principale, mais où plusieurs idées et émotions s'additionnent et complexifient l'énoncé. La bannière anticipe ainsi sur un lecteur prêt à se laisser convaincre par l'addition entre les potentiels d'action du texte et du mouvement.

Les images fixes juxtaposées aux animations textuelles renvoient aux joies des vacances du ski. Les couplages entre textes et images peuvent en revanche rappeler le caractère éphémère de ces « visions » pour celui qui ne profite pas de la promotion.

\section{Figures de la lecture « pro-immersive »}

En créant des ambiances, sensations et émotions déjà expérimentées ailleurs, certains couplages entre texte et mouvements préfigurent un lecteur prêt à s'immerger au moins momentanément dans un texte devenu « simulacre de référent».

Les noms des parfums Kenzo sont animés sur le site de la marque comme s'ils s'approchaient du lecteur, avant de passer vers le horschamp sans perdre en intensité lumineuse. Ces « trajectoires » renvoient potentiellement à des idées de « cohérence » et de « persistance ». Aux noms de parfum, le lecteur associe potentiellement des idées d'odeur, d'expérience sensuelle, peut-être d'agrément.

Le couplage entre mots et mouvements peut d'abord être appelé « additionnel », anticipant sur l'idée d'un parfum persistant. Le mouvement continu des noms surgissant du centre de l'espace dédié à l'annonce, pourrait également faire penser à l'action d'un diffuseur de parfums qui ne s'épuise jamais. Mots et mouvement formeraient ainsi un procédé rhétorique que je propose d'appeler " ciné-gramme », en hommage au calligramme dans l'univers papier. En effleurant les mots, le lecteur peut orienter ce « nuage d'odeurs »; en les activant, il plonge dans l'ambiance associée. L'énoncé prononcé par la voix de femme, « Il était une fois... Kenzo », soutient l'invitation à des pratiques de lecture qui me paraissent être de l'ordre de l'immersion. 
Sur la bande-son, des pas s'approchent et s'éloignent. Ne dit-on pas qu'un parfum laisse un « sillage », qui nous rappelle son porteur/ sa porteuse même si la personne n'est plus présente ? Ce sillage laisset-il des traces mnésiques qui nous ramènent vers l'enfance - le temps des « il était une fois »? Le " pouvoir » des parfums Kenzo résiderait donc non seulement dans leur persistance, mais dans les traces qu'ils laissent dans notre mémoire en nous plongeant, encore et encore, dans une parenthèse enchantée.

\section{Conclusion}

Les « couplages » entre textes et mouvements dans les bannières analysées anticipent sur des pratiques de lecture qui s'actualisent de façon plus ou moins complète dans une situation de réception. La rhétorique de la réception identifie les procédés rhétoriques formés par ces couplages et énonce des hypothèses sur la rencontre possible entre ces procédés et l'horizon d'attente potentiel du lecteur. Le créateur d'une animation textuelle publicitaire ne peut pas compter sur le fait que le lecteur y prête une attention consciente. Couplant des textes à des mouvements à caractère « iconique », il préfigure néanmoins plusieurs pratiques de lecture possibles, dont la lecture intensive. Entre textes et mouvements, il crée des «figures de la lecture » dont certaines ont été identifiées et nommées dans cet article.

La méthodologie et les résultats de cette étude sémio-rhétorique du texte numérique renvoient aux problématiques d'une " sémiotique du sensible ", mise en œuvre depuis plusieurs années par des auteurs comme Jean-Jacques Boutaud (2007) et Eric Landowski (2004) dans d'autres domaines. Pour Eric Landowski, le sensible est un élément important dans tout acte de communication. Jean-Jacques Boutaud (2007) précise que le rôle du sensible a cependant encore gagné en importance dans un monde où «le sens de l'expérience est guidé par la recherche de sensations, par l'immersion dans des contextes enveloppants, polysensoriels ». Le " régime dominant " actuel est, selon l'auteur " celui de l'esthésie et des émotions sensorielles favorisées par synesthésie, par coopération des sens ». Ces observations me paraissent primordiales pour cerner le caractère plurisémiotique du texte numérique. "Savons-nous prendre en compte la part sensible du sens ? », se demande Jean-Jacques Boutaud (2007). J'espère que la démarche d'analyse et la terminologie dont cet article présente des extraits, permettra de circonscrire les conditions d'émergence de ces 
« synesthésies » dans de nombreux exemples de textes numériques même s'il peut toujours sembler problématique de circonscrire des sensations et émotions par des mots.

\section{Références}

Boutaud, J.-J. (2007). « Du sens, des sens. Sémiotique, marketing et communication en terrain sensible ». Semen 23, http://semen.revues.org/5011 (consulté le 11 février 2012)

Courbet, D. (2004). Communication médiatique : les apports de la psychologie sociale. Pour une pluralité épistémologique, théorique et méthodologique en SIC. Note de synthèse des travaux pour l'obtention de l'Habilitation à Diriger des Recherches en Sciences de l'information et de la communication. Université de Provence-AixMarseille 1.

Courbet, D., Borde, A., Intartaglia, J., \& Denis, S. (2004). « L'influence non consciente des publicités vues furtivement et aussitôt oubliées : une méthode d'étude sociocognitive appliquée à internet ». Questions de communication, 5, p. 83-102.

Fish, S. (1980). Is There a Text in This Class? The Authority of Interpretative Communities. Cambridge : Harvard University Press.

Frey, A., Poitrenaud, S., \& Tijus, C. (2010). « Validation expérimentale de la pertinence cognitive des UST ». Musimediane, 5 (2010), http://www.musimediane.com/ (consulté le 18 juin 2012).

Iser, W. (1976 ; 1995). L'Acte de lecture - Théorie de l'effet esthétique. Paris : Mardaga.

Jauss, H. R. (1972-1978; 1978 ; 2010). Pour une esthétique de la réception. Paris : Gallimard.

Jeanneret, Y. (2007). Y a-t-il (vraiment) des technologies de l'information ? Paris : Presses Universitaires du Septentrion.

Jeanneret, Y., \& Souchier, E. (2005). «L'énonciation éditoriale dans les écrits d'écran ». Communication et langages, 145, p. 3-15.

Kapferer, J.-N., Laurent, G. (1986). "Les profils d'implication ». Recherche et Applications en Marketing, 1, p. 3-20.

Klinkenberg, J.-M. (1996). Précis de sémiotique générale. Bruxelles : De Boeck Université.

Landowski, E. (2004). Passions sans nom. Paris : PUF.

Meunier, J.-P. (2006). «Pour une approche cognitive de la signification iconique ». Bernard Darras (dir.). Images et sémiotique: Sémiotique pragmatique et cognitive. Paris : Publications de la Sorbonne, p. 133-145.

Perelman, C. (1977 ; 2000), L'Empire rhétorique - Rhétorique et argumentation. Paris : Vrin.

Pignier, N. (2005). « Analyse sémiotique de la webpublicité ». Semiotica, 157(1/4), p. 521-538.

Shapiro, S., \& Krishnan, H. S. (2001). « Memory-based Measures for Assessing Advertising Effects: A Comparison of Explicit and Implicit Memory Effects ». The Journal of Advertising, 30 (Fall), p. 1-14.

Souchier, E. (1998). «L'image du texte - Pour une théorie de l'énonciation éditoriale ». Dans : Les cahiers de médiologie, 6, p. 137-145. 